\title{
A Multimodal Discourse Analysis on the Reports of China's First Gold: A Comparative Study of BBC and VOA
}

\author{
Liukexin Cheng
}

\author{
School of English Studies, Xi'an International Studies University \\ E-mail: chengliukexin@qq.com
}

\begin{abstract}
Finding that there are little comparative studies of multimodal discourses, this research intends to provide a case study of this field, making a comparison and contrast between two multimodal discourses and analyzing their attitudes through their visual designs and recontextualizations. Recently, a Chinese girl, Yang Qian won the first gold medal in Tokyo Olympic competition. This news is reported by large numbers of mass media around the world. All kinds of presses contain various attitudes and ideologies. Therefore, the reports from BBC and VOA are chosen to make a comparative MDA study, estimating their attitudes toward China's first gold.
\end{abstract}

Keywords: Comparative study of multimodal discourse analysis, Visual grammar, Recontextualization, Tokyo Olympic game.

\section{INTRODUCTION}

With the proliferation of multimedia, there are plenty of modes and resources conveying people's opinions and thoughts. Multimodal discourse analysis equips people the ability to figure out the ideologies behind the contexts. There are many researches and studies in this field. There is a review on previous researches about the visual grammar, recontextualization and the multimodal discourse analysis.

In 2004, M.A.K. Halliday lays the foundation of functional grammar with the theory of meta-functions of language [1]. With the reference to Halliday's theory, Theo Van Leeuwen and Gunther Kress put forward the theoretical framework in the field of visual grammar [2]. The visual grammar mainly has three meanings: the representational meaning, the interactive meaning and the compositional meaning. In 2008, Van Leeuwen explains the representational meaning specifically [3].

In 1993, Van Leeuwen states that the discursive strategy relies on the ideology behind the news [4]. In 2008, he introduces the recontextualization of social practice: substitutions, deletions, rearrangements, additions which includes repetitions, reactions, purposes, legitimations, and evaluation [3]. This theory is applied in plenty of researches. For instance, in 2016,
Wei Zhongsheng analyzes the news translation strategies under the perspective of recontextualization [5]. He also mentions the doubted faithfulness of the news.

In 1977, Roland Barthes realizes the connection between the image and our language (qtd. in Zhang Delu 24) [6]. In 2001, Kress and Van Leeuwen provide a detailed information about the design of contemporary multimedia interaction [7]. In 2012, David Machin and Andrea Mayr provide a systematic introduction to the MDA in plenty of aspects [8]. In 2003, Zhanzi Li illustrates the application of MDA to semiotics [9]. And Zhang Delu makes an improvement of it under the perspective of systemic-functional theory in 2018 [10]. In the same year, Li Cunjie analyzes the multimodal discourses about China on The Economist, demonstrating how the mass media's ideologies influence readers through their intended verbal or visual choices [11].

In 2016, Liu Zhangting provides a case study of the comparative multimodal discourse analysis [12]. However, comparative MDA are in minority. Therefore, the research intends to fill this void, using the approach of visual grammar and the theory of recontextualization to estimate the multimodal media discourses from BBC and VOA respectively and then evaluate whether their attitudes toward China's first gold in Tokyo Olympic 
game are the same.

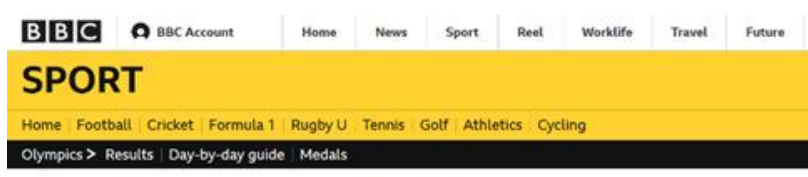

Tokyo Olympics: First gold of Games goes to China's Yang Qian in shooting

O2A sus 2021 ormpics

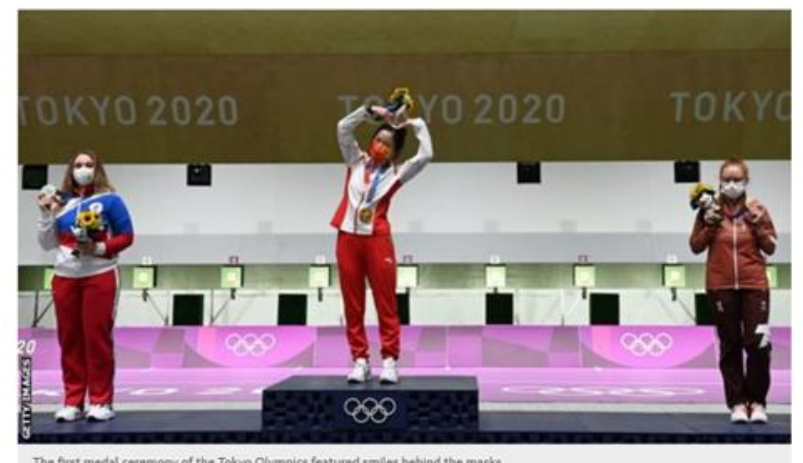

Tokyo Olympik Games on the BAC

Dates: 23 July-8 August Time in Tokyo: BST +8

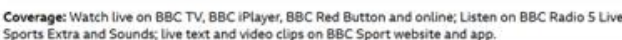

She had to put her own medal round her neck and sing the national anthem behind a mask, but Chinese shooter Yang aian still celebrated winning the first gold medal of the Toky

The morning after the delayed Games had opened with a scaled-back and sombre ceremony. Yang won the 10-metre rifle competition in front of empty stands.

She was handed her medal on a tray by International Olympic Committee president Thomas Bach in accordance with the Covid-19 protocols that will affect every aspect of these Games. Rock music had been piped into the venue and only a handful of Chinese support staff were there to congratulate her on her victory after a nail-biting final in which she clinched victory with an Olympic
moment

ITm extremely proud and happy to win," said Yang, 21.

Figure 1 The web page of the BBC report [13]

\section{MULTIMODAL DISCOURSE ANALYSIS OF TWO REPORTS}

\subsection{Analysis of BBC Report}

\subsubsection{Visual Grammar}

\subsubsection{Interactive Meaning}

Although the pixel is not high, people can still have the eye contact with Yang, sharing her golden happiness. Because Yang is wearing a mask, her facial expression is hardly demonstrated. But Yang Qian uses her gesture to express her delight. The photo is taken from a fontal angle, creating a sense of involvement for the viewer. A sense of equality is created from an equal angle. The medium shot enables viewer to observe the body gesture of Yang and includes the other two athletes beside her, implicating Yang's special and higher position. White, orange and light purple constitutes the background. Yang wears red and white clothes. The dark blue stage reveals Yang Qian's determination. Every athlete holds a bunch of sun flower. The whole image is bright and vibrant, conveying positive attitude.

\subsubsection{Compositional Meaning}

The report is read from top to bottom. The information is from real to ideal and then to real. Yang is standing in the middle of the whole picture. The information here is from center to margin. Yang is highlighted through the focus of the light. And other two athletes have darker clothes. Viewer can immediately recognize who is the winner.

\subsubsection{Representational Meaning}

In the photo, Yang Qian is bending and rounding her arms to shape a sweet heart. This image is a narrative image with vectors. Yang is standing on the middle of the podium, holding the sun flower. She is the most significant figure in the image and the brightest one. Her action expresses her positive feeling, also causing viewer to wonder who is she forming to. The Olympic rings is under her feet, representing her success in the game.

\subsubsection{Recontextualization}

At the beginning, the headline demonstrates the winner clearly. The order of the time is rearranged. Reader can see the result of the competition firstly. The attention of the reader is attracted. However, the focus of Yang transmits to the pandemic in the subtitle under the image. Additionally, the text emphasizes that Yang has the medal by her own and sing the national anthem with the mask. Yang is generalized when compared with the "mask". Her actions are related to the COVID-19: the ceremony is simplified so that Yang only stands on an empty podium; she gets the medal through a tray because individuals is required not to approach; only few Chinese people celebrates her victory in the stadium because the stadium is not allowed to hold too many people. The pandemic repeats many times in the text.

\subsection{Analysis of $V O A$ Report}

\subsubsection{Visual Grammar}




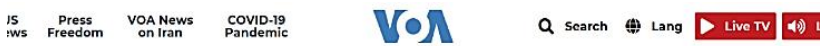

\section{First Gold Medals, First Exits of Tokyo Games}

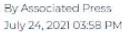

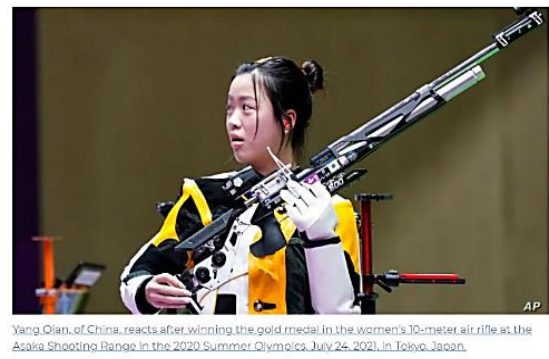

TOKYO - Hardly half a day passed before politics, the pandemic and blistering heat impacted events across the Tokyo Olympics.

China's Yang Qian, at least, stayed right on target.

Yang overtook Anastasilia Galashina of Russia to win the first gold medal of the Tokyo Games in the women's 10-meter air rifle Saturday. Her last shot was her worst of the finals but still rallied her past Galashina with an Olympic-record score of 251.8. Switzerland's Nina Christen took bronze

"It's unbelievable that I can be here," Yang said through an interpreter. "I was really nervous. The competition was really tight, but I'm so happy that I could win."

Galashina led Yang by 0.2 point when they fired almost simultaneously on their last shots. The limited crowd at Asaka Shooting Range let out gasps as the scores posted a split second later.

Yang, 21, who qualified sixth of the eight medal competitors, missed the innermost circle on her final shot, a 9.8 that she figured would cost her gold.

She looked up to see Galashina had missed the two center rings. The Russian's 8.9 meant IOC President Thomas Bach would present Yang the gold medal on a tray per pandemic protocols - instead of Galashina

Figure 2 The web page of the VOA report [14]

\subsubsection{Interactive Meaning}

In the picture, Yang has no eye contact with the viewer. The distance appears in this offer image. Opening her mouth slightly, Yang's facial expression demonstrates her tension and exhaustion. Her hair covers her face because she does not have chance to settle it. Yang's picture is taken from an oblique angle, making the viewer feels a sense of exclusion and detachment. Equal angle implicates that Yang has the same social power with the viewer. Also, this image is captured in a close shot. There is an intimate distance between Yang and the viewer. The background color is brown. Excepting little orange on Yang's clothes and the rifle, most of the color around Yang is black and white. There is no colorful object. The atmosphere of tension is created. Moreover, the whole background of the web page is white. There is no decoration or other color in the main text.

\subsubsection{Compositional Meaning}

Yang's long rifle separates the picture into two parts from lower left to upper right. Viewer can see Yang's tension and uncertainty firstly and then find her rifle. The orientation of her facial expression and her gesture compose a hold vector. The release vector and the hold vector achieve the Yin Yang balance of the whole picture. In the portrait, Yang's facial expression and gesture are in the middle of the image. The background is designed to be vague. The information of the web page is from top to the bottom-from real to ideal and to real again.

\subsubsection{Representational Meaning}

The portrait is a narrative image with dynamic processes. Yang is holding her rifle and looking at somewhere. Viewer consequently has some reactions on this - wondering who is she looking at. This narrative process draws viewer's attention and encourages viewer to continue the reading. Her clothes and the rifle implicate her shooter identity. The rifle and its stand form a solemn atmosphere.

\subsubsection{Recontextualization}

The headline does not provide the main participant of the event. This generalizes Yang Qian's victory and on the other hand, attracts reader's attention. The subtitle then illustrates Yang's winning concisely.

But the beginning of the report does not focus on Yang Qian directly. The pandemic, political and climate impacts are shown. The contrast between Yang and her competitor Galashina is repeated, showing the intensity of the competition. Providing Yang's previous record, the text implicates that Yang's victory is not definite. Yang's remarks implicate her lack of self-confidence. Moreover, the text does not emphasize Yang Qian's awarding process. Reader is excluded from sharing Yang's happiness of success. The distance between Yang and viewer is created.

\section{COMPARISON AND CONTRAST}

\subsection{Visual Grammar}

Table 1 The comparison and contrast of the visual grammar between two reports

\begin{tabular}{|c|c|c|c|}
\hline Three meanings & & BBC report & VOA report \\
\hline \multirow[t]{6}{*}{$\begin{array}{l}\text { Interactive } \\
\text { meaning }\end{array}$} & $\begin{array}{l}\text { Social } \\
\text { interaction }\end{array}$ & Demand & Offer \\
\hline & $\begin{array}{l}\text { Facial } \\
\text { expression/sta } \\
\text { nce }\end{array}$ & Positive & Negative \\
\hline & Horizontal & Frontal & Oblique \\
\hline & $\begin{array}{l}\text { Social } \\
\text { distance }\end{array}$ & Medium & Close \\
\hline & Vertical & Equal & Equal \\
\hline & Web page & colorful & simplified \\
\hline $\begin{array}{l}\text { Compositional } \\
\text { meaning }\end{array}$ & $\begin{array}{l}\text { Information } \\
\text { value }\end{array}$ & $\begin{array}{l}\text { Center- } \\
\text { margin }\end{array}$ & YinYang \\
\hline
\end{tabular}




\begin{tabular}{|l|l|l|l|}
\hline $\begin{array}{l}\text { Representational } \\
\text { meaning }\end{array}$ & Narrative & Narrative \\
\hline
\end{tabular}

As is shown in table 1, both of the images put the focus on Yang Qian through their respective framing. BBC's image demonstrates Yang's happiness and her higher position. VOA's image portrays an exhausted and nervous athlete holding her rifle without the happiness of success. According to the two images, $\mathrm{BBC}$ and VOA have different attitudes toward Yang Qian.

\subsection{Recontextualization}

Two discourses both applies the transformation to realize their representations of the social practice. In both articles, the focus is transformed from Yang Qian to other things. Yang Qian's first gold is not of the most significance in two reports. BBC integrates pandemic impact into the event, leading the viewer to consider the negative global situation rather than simply celebrating Yang Qian's victory. VOA stresses the vehemence of the competition. By quoting Yang's words, VOA shows her nervousness. Both of them append the influence of the COVID-19 in the first gold of the Tokyo Olympic games. Both reports generalize Yang Qian's victory.

\section{CONCLUSION}

Through the comparative analysis, the attitudes of the reports from $\mathrm{BBC}$ and $\mathrm{VOA}$ are gradually clear. BBC's image delivers much more positive information about Yang Qian than that in VOA. While in the text, both the reports transform the participant to other elements of the social practice to locate their points. As prominent media, BBC and VOA hold different points of view and applies diverse techniques to influence the readers and create their expected reactions. This study has a pragmatical implication for the field of multimodal discourse analysis. And the research illustrates that the attitude and the ideology behind the discourse matters.

\section{REFERENCES}

[1] Halliday, M. A. K., \& Matthiessen, C. M. I.. (2004). An Introduction to Functional Grammar (3rd ed.). Hodder Arnold.

[2] Kress, G., \& Van Leeuwen, T. (2006). Reading Images: The Grammar of Visual Design (2nd ed.). Routledge.

[3] Van Leeuwen, T. (2008). Discourse and Practice: New Tools for Critical Discourse Analysis. Oxford University Press.

[4] Van Leeuwen, T. (1993). Genre and field in critical discourse analysis: a synopsis. Discourse \& Society, 4(2): 193-223. DOI : https://doi.org/10.1177/0957926593004002004

[5] Wei, Z. (2016). Recontextualization of News Discourse and Its Translation from Multiple Perspectives. Journal of University of Shanghai for Science and Technology, 38(1): 16-20,92. DOI : https://doi.org/10.13256/j.cnki.jusst.sse.2016.01.00 4

[6] Zhang, D. (2009). On A Synthetic Theoretical Framework for Multimodal Discourse Analysis. Foreign Languages in China, 6(1): 24-30. DOI : https://doi.org/10.13564/j.cnki.issn.16729382.2009.01.004

[7] Kress, G., \& Van Leeuwen, T. (2001). Multimodal Discourse : The Modes and Media of Contemporary Communication. In Hodder Arnold. Bloomsbury Academic. DOI : https://doi.org/10.1017/cbo9781139567701.010

[8] Machin, D., \& Mayr, A. (2012). How to Do Critical Discourse Analysis: A Multimodal Introduction. SAGE Publications Ltd.

[9] Li, Z. (2003). Social Semiotic Approach to Multimodal Discourse. Foreign Languages Research, No.5. DOI : https://doi.org/10.13978/j.cnki.wyyj.2003.05.001

[10] Zhang, D. (2018). A Systemic-Functional Synthetic Framework for Multimodal Discourse Analysis. Modern Foreign Languages, 41(6): 731-743.

[11] Li, C. (2018). A Multimodal Discourse Analysis of China-related Cover on The Economist. Youth Journalist, 21:89-90. DOI : https://doi.org/10.15997/j.cnki.qnjz.2018.21.049

[12] Liu, Z. (2016). A Multimodal Discourse Analysis of Chinses and American Animation Film Posters-Monkey King: Hero is Back and Kung Fu Panda 2. Overseas English, 3: 203-204,208.

[13] Tokyo Olympics: First gold of Games goes to China's Yang Qian in shooting. (2021, July 24). Retrieved from https://www.bbc.com/sport/olympics/57952436.

[14] Associated Press. (2021, July 24). First Gold Medals, First Exits of Tokyo Games. Retrieved from https://www.voanews.com/tokyoolympics/first-gold-medals-first-exits-tokyogames. 